\title{
NON UNIFORMITY EXPECTED IN NOVA EJECTION
}

\author{
M. FRIEDJUNG \\ INSTITUT D'ASTROPHYSIQUE \\ DE PARIS - C.N.R.S. \\ 98 BIS BD ARAGO \\ F 75014 PARIS
}

\section{Summary}

It is shown that according to the model of continued ejection of nova envelopes by radiation pressure, discrete clouds are probably formed. Their properties are discussed.

I suggested a few years ago, that contined ejection of a nova after its visual maximum, might be in discrete slabs (Friedjung 1975, 1977). Such a form of continued ejection would tend to give the same photospheric radii in these phases, for a smaller mass flux, than if the ejection was completely uniform. The mass ejected in these phases would then be less with respect to that of the principal system ejected near maximum light, which seems to contain most of the mass of the envelope.

The question that can be asked is whether such an effect can be justified in the framework of radiation pressure driven wind models (Friedjung, 1966, Bath and Shaviv, 1976), which seem best able to account for many features of novae, and which now are more fashionable than some years ago. For such a wind to occur the central star must have a luminosity above the Eddington limit, which if this occured throughout the star would render it unstable. This difficulty could be overcome if spherical symmetry did not exist, and the high luminosity were produced intermittently in localized regions. Now an estimate I made for uniform models (Friedjung, 1966) suggests that in the regions where ejection takes place, the photon diffusion velocity is, unlike in layers further out, not faster than the gas velocity. In such a situation photons can be confined in bubbles. The production of such bubbles as a result of very temperature sensitive processes of energy generation (e.8. nuclear) is quite conceivable; radiation pressure would tend to expel the gas within them in the region where ejection occurs, where radiation pressure would considerably exceed the gas pressure. The gas would thus be concentrated into seperate clouds between the expanding bubbles. These ideas inspired by the comments of Shaviv (1977), suggest a process which can be compared to boiling.

Without at this stage studying the detailed physics of the bubbles it is possible to make quantitative estimates concerning the ejected clouds. Firstly one can estimate the minimum dimensions of the clouds if one notes that no uniform solution of motion through the critical point can be found, when the hydrodymamical 
equations are examined. This problem does not exist if discrete clouds are ejected. According to the ideas suggested here, one could expect discrete clouds to be produced when the bubbles merged, it being reasonable to consider the layer where this occured as that where the clouds started to be accelerated. The clouds would then expand at about 3 times the sound speed perpendicular to the direction of acceleration, and a consideration of the equation of motion of a cloud to be discussed below suggests that at the critical point, the cloud could not be smaller than about 10 times the height of this point above the layer where acceleration started. At the critical point the clouds must remain seperate and not merge to avoid the difficulty just mentioned of uniform models. One can therefore estimate the minimum diameter of a cloud at this point as about $10^{-3}$ of the radius of the region where acceleration starts and a maximum diameter of the order of this radius. Also in order for clouds not be destroyed by collisions with clouds not ejected in exactly the same direction below the critical point (different perts of a cloud would then undergo different collisions), only about one cloud can be supposed to exist in a given direction below the critical point at any time.

In order to see what happens to such clouds the equation of motion needs to be considered. The same equations as those of the uniform case can be used, with a few differences. When the density $\rho$ is set equal to $\frac{\mathrm{m}}{\mathrm{vr}^{2}}$ with $\mathrm{v}$ the velocity and $r$ the distance from the centre of the ejecting star, m is" no longer rearly constant, but varies from the centre to the edge of a cloud. The radiation field is no longer quite uniform. This latter lack of uniformity will be neglected here except within a cloud. One can justify this, as most accelaration takes place over a distance of the order of the radius of the ejecting region, and over most of this distance a cloud may receive a mean of radiation from many clouds and bubbles. It is also assumed that unlike in lower layers radiation diffuses faster than the expansion velocity (assumption discussed in Friedjung, (1966). Using equations (7), (8) and (32) of Friedjung (1966), one obtains for a cloud putting $\rho=\frac{\bar{m}}{\mathrm{vr}^{2}}$ (in is an average of $\mathrm{m}$ from radiating surface to cloud),

$$
\begin{aligned}
\frac{d\left(v^{2}\right)}{d r}=\left(\frac{2}{c}(K+\sigma) F_{c}\right. & -2 G M) \frac{1}{r^{2}}-\left(\frac{k+\sigma}{c}\right) \frac{\bar{m}}{r^{2}}\left[v^{2}+2 G M_{*}\left(\frac{1}{r *}-\frac{1}{r}\right)\right] \\
& + \text { negligible gas pressure terms } \ldots \ldots \ldots
\end{aligned}
$$

Here $K$ is the opacity due to free-bound + free-free absorption, and $\sigma$ that due to electron scattering. $F_{c}$ is the total radiant flux over one radian of the ejecting star before absorption by the envelope, while $M_{*}$ and $r_{*}$ are the ejecting star's mass and radius respectively. In the derivation of equation (1), equation (32) of Friedjung (1966) has been corrected to take account of the conversion of some radiant energy into gravitational potential energy.

One can see what happens to an individual cloud if one considers the second term of equation (1). This term occurs because some of the radiation flux is conver- 
ted into kinetic and gravitational potential energy. If one considers different parts of one cloud, it is not constant as less radiation reaches the back (further from the ejecting surface) before being converted. Thus the term increases from the front to the back of the cloud, and becomes

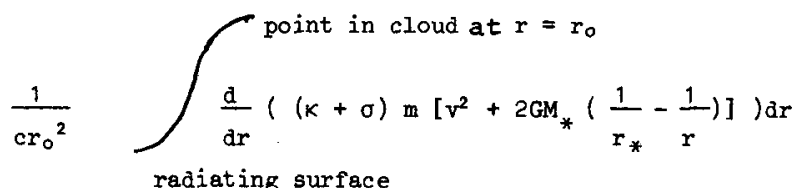

If $\sigma \gg k$ as expected in the uniform case (Bath and Shaviv, 1976), the front of a cloud is more accelerated than its back, and the cloud tends to be compressed to a thin sheet; a cloud is then therefore stable in this case. If $K \gg \sigma$ however, $\kappa+\sigma$ is proportional to $\rho T-{ }^{3} \cdot{ }^{5}$ with $T$ the temperature. The front of the cloud would be hotter than the back, and the back (as well as higher density central parts of the cloud if they existed) would be accelerated away from the front as the first term in equation (1) is larger than the second one. A cloud in this second case, would not be stable. As a result of these considerations one can expect such a cloud and indeed any large inhomogeneity to be compressed in the direction of motion till $k \simeq \sigma$. The compression to $k \simeq \sigma$ would not much change the ratio of the radiation pressure gradient to the gas pressure. However the variation of the second term of equation (1) would be less across a smaller cloud, and the gas pressure would stop a cloud being compressed to a slab, if it contained less than $10^{-3}$ of the mass of gas in a given direction from the ejecting surface to the cloud.

Acceleration in models of the type discussed here, occurs at very large optical depths $\left(\simeq 10^{2}\right)$, and the photosphere (where the optical depth is of order unity, and where the deepest layers accessible to direct observation are located) is much further from the ejecting star. Before reaching the photosphere the clouds would undergo collisions both before and after acceleration. The maximum relative velocity

of colliding clouds (or parts of clouds) is of the order of the ejection velocity times $\frac{r_{*}}{r}$, as clouds with larger relative velocities, if they existed, would not survive as separate clouds to $x$. When one compares the rate of free-free cooling proportional to $\rho^{2} \mathrm{~T}^{1 / 2}$ and the energy liberated in a collision proportional to $\rho v^{2}$ relative, one finds that the time required for cooling would be considerably less than that for all the gas of one slab to be braked by the gas of another. It seems therefore justified to neglect non radiative heating by collisions below the photosphere.

Approximate calculations of the number of clouds in the photosphere after collisions and coalescence in one line of sight, have been made. It was supposed that both the cloud $\bar{m}$ 's, and the intervals between the departure times from the acceleration region in a given direction were random between zero and a constant. The departure velocity from the acceleration region was obtained by integrating equa- 
tion (1) to infinity, which gives:

$$
v_{\infty}^{2}=\frac{2 F c}{\bar{m}}\left(1-e^{-\left(\frac{\sigma}{c} \frac{\bar{m}}{r}\right)}\right)-2 \frac{G M}{r_{*}}
$$

This equation for a single cloud does not take account of collisions in the acceleration region, and thus only can be used to give an indication of the effects to be expected. In trial calculations performed 25 times $\frac{\sigma}{c} \frac{\bar{m}}{r_{*}} \max$ was taken as 1.5 and $\frac{G M}{r_{*}} \frac{\bar{m}}{\overline{F C}} \max$ as 0.5 . After the ejection of 99 clouds, an average of $24 \pm 10$ clouds occured at $r^{\prime} s$ of more than half that of the outemost cloud, instead of tne 50 expected if no collisions took place. A more realistic calculation performed 7 times in which 494 clouds were ejected gave an average of $46 \pm 25$ clouds.

The conditions for cloud stability above the acceleration region are the same as those vithin it. Assuming that in the photosphere, the temperature is $3 \times 10^{4}$, the density $1 / 2 \times 10^{-11}$, and the mean velocity $1 \times 10^{3} \mathrm{~km} / \mathrm{sec}$, the $\sigma \simeq k$ condition is satisfied for slabs compressed by a factor of $10^{3}$. The variation of the second term of equation (1) across a slab would decrease with respect to the gas pressure as $r$ increased (the ratio varying nearly as $1 / r$ ). If the photosphere occured at $10^{2} r_{*}$, the clouds whose number in one direction would have been reduced by collisions would still be almost stable with respect to disruption by the gas pressure. Different parts of clouds would undergo different collisions, unless clouds were always ejected in the same directions. They would thus tend to be broken up, but the different pieces would still remain thin slabs. This calculation is very approximate, but it seems that in many cases slab like clouds could survive till they reached the photosphere, without needing other forces to hold them together.

Rapid time variations over time scales of $10^{3}-10^{4}$ sec in the photosphere might be expected from the present model because of random variation of mean cloud properties. These variations would however be very small, unless clouds were always ejected in the same directions and not broken up by collisions.

The ideas here presented are preliminary and need to be checked by detailed calculation. If true, novae would have continued but not continuous ejection. The processes described may not only be applicable to novae, but also to other types of extremely unstable star with hot winds which are optically thick in the continuum. Thus nova like processes may occur for certain symbiotic stars (Bath, 1977), objects like $n$ Carinse and even certain Wolf Rayet stars. 
Bath,G.T. : 1977, Mon. Not. R. Astr. Soe. 178, 203

Bath,G.T., Shaviv,G. : 1976, Mon. Not. R. Astr. Soc. 175, 305

Friedjung,M. : 1966, Mon. Not. R. Astr. Soc. 132, 317

Friedjung,M. : 1974, in "Variable Stars and stellar Evolution" ed. Sherwood and Plaid, IAU Symp. 67, p.335

Friedjung,M. : 1977, in "Novze and Related Stars" Ed. Friedjung, Reidel, Dordrecht,

Shaviv,G. : 1977, in "Novae and Related Stars" Ed. Friedjung, Reidel, Dordrecht, p. 223 